\title{
ABM Clinical Protocol \#1: Guidelines for Blood Glucose Monitoring and Treatment of Hypoglycemia in Term and Late-Preterm Neonates, Revised 2014
}

\author{
Nancy Wight, ${ }^{1,2}$ Kathleen A. Marinelli, ${ }^{3,4}$ and The Academy of Breastfeeding Medicine
}

A central goal of The Academy of Breastfeeding Medicine is the development of clinical protocols for managing common medical problems that may impact breastfeeding success. These protocols serve only as guidelines for the care of breastfeeding mothers and infants and do not delineate an exclusive course of treatment or serve as standards of medical care. Variations in treatment may be appropriate according to the needs of an individual patient.

\section{Purpose}

T

- Prevent clinically significant hypoglycemia in infants

- Appropriately monitor blood glucose levels in at-risk term and late-preterm infants

- Manage documented hypoglycemia in infants

- Establish and preserve maternal milk supply during medically necessary supplementation for hypoglycemia or during separation of mother and baby

\section{Background}

\section{Physiology}

The term "hypoglycemia" refers to a low blood glucose concentration. Clinically significant neonatal hypoglycemia reflects an imbalance between the supply and utilization of glucose and alternative fuels and may result from several disturbed regulatory mechanisms. ${ }^{1}$ Transient hypoglycemia in the first hours after birth is common, occurring in almost all mammalian newborns. In healthy, term human infants, even if early enteral feeding is withheld, this phenomenon is self-limited, without clinical signs, and considered to be part of adaptation to postnatal life, as glucose levels spontaneously rise within the first 24 hours after birth (for some, it is even longer but still physiological) ${ }^{2-6}$ Most neonates compensate for this "physiological" low blood glucose with endogenous fuel production through gluconeogenesis, glycogenolysis, and ketogenesis, collectively called "counter-regulation." Even in those situations where low blood glucose concentrations do develop secondary to prolonged intervals ( $>8$ hours) between breastfeeding, a marked ketogenic response occurs. The enhanced capability of the neonatal brain to utilize ketone bodies provides glucose-sparing fuel to the brain, protecting neurological function. ${ }^{3,7-9}$ The compensatory provision of alternate fuels constitutes a normal adaptive response to transiently low nutrient intake during the establishment of breastfeeding, ${ }^{3,10}$ resulting in most breastfed infants tolerating lower plasma glucose levels without any significant clinical manifestations or sequelae. ${ }^{10}$

No studies have shown that treating transiently low blood glucose levels results in better short-term or long-term outcomes compared with no treatment, and in fact there is no evidence at all that hypoglycemic infants with no clinical signs benefit from treatment. ${ }^{11,12}$ Increases in neurodevelopmental abnormalities have been found in infants who have hypoglycemia associated with abnormal clinical signs, especially those with severe, persistent hyperinsulinemic hypoglycemia. ${ }^{11-16}$ Rozance and Hay ${ }^{17}$ have delineated the conditions that should be present before considering that long-term neurologic impairment might be related to neonatal hypoglycemia. Transient, single, brief periods of hypoglycemia are unlikely to cause permanent neurologic damage. ${ }^{18-21}$ Therefore, the monitoring of blood glucose concentrations in healthy, term, appropriately grown neonates is unnecessary and potentially harmful to parental wellbeing and the successful establishment of breastfeeding. ${ }^{18-23}$

\section{Definition of hypoglycemia}

The definition of hypoglycemia in the newborn infant has remained controversial because of a lack of significant

\footnotetext{
${ }^{1}$ San Diego Neonatology, Inc., San Diego, California.

${ }^{2}$ Sharp HealthCare Lactation Services, Sharp Mary Birch Hospital for Women and Newborns, San Diego, California.

${ }^{3}$ Division of Neonatology and The Connecticut Human Milk Research Center, Connecticut Children's Medical Center, Hartford, Connecticut.

${ }^{4}$ University of Connecticut School of Medicine, Farmington, Connecticut.
} 
Table 1. Population Low Thresholds: Plasma Glucose Level $^{40}$

\begin{tabular}{lc}
$\begin{array}{l}\text { Hour(s) after } \\
\text { birth }\end{array}$ & $\leq 5^{\text {th }} \begin{array}{c}\text { percentile plasma } \\
\text { glucose level }\end{array}$ \\
\hline $1-2$ (nadir) & $28 \mathrm{mg} / \mathrm{dL}(1.6 \mathrm{mmol} / \mathrm{L})$ \\
$3-47$ & $40 \mathrm{mg} / \mathrm{dL}(2.2 / \mathrm{mmol} / \mathrm{L})$ \\
$48-72$ & $48 \mathrm{mg} / \mathrm{dL}(2.7 \mathrm{mmol} / \mathrm{L})$ \\
\hline
\end{tabular}

correlation among plasma glucose concentration, clinical signs, and long-term sequelae. ${ }^{10,24,25}$ An expert panel convened in 2008 by the U.S. National Institutes of Health concluded that there has been no substantial evidence-based progress in defining what constitutes clinically important neonatal hypoglycemia, particularly regarding how it relates to brain injury. ${ }^{26}$ Multiple reviews have concluded that there is no specific plasma or blood glucose concentration or duration of low blood glucose level that can be linked to either clinical signs or permanent neurologic injury. ${ }^{17,25,27}$ In addition, blood glucose test results vary enormously with the source of the blood sample, the assay method, and whether whole blood, plasma, or serum glucose concentration is determined. Plasma or serum glucose concentrations are $10-15 \%$ higher than in whole blood. ${ }^{28,29}$

Breastfed, formula-fed, and mixed-fed infants follow the same pattern of glucose values, with an initial fall inglucose level over the first 2 hours of life, followed by a gradual rise in glucose level over the next 96 hours, whether fed or not. ${ }^{2,5,6}$ Artificially fedinfantstend tohave slightlyhigher levels of glucose and lower levels of ketone bodies than breastfed infants. ${ }^{3,5,18,30-32}$

The incidence of "hypoglycemia" varies with the definition. ${ }^{33,34}$ Many authors have suggested numeric definitions of hypoglycemia, usually between 30 and $50 \mathrm{mg} / \mathrm{dL}$ $(1.7-2.8 \mathrm{mmol} / \mathrm{L})$ and varying by postnatal age., $, 5,18,24,26,33,35-38$ There is no scientific justification for the value of $<47 \mathrm{mg} / \mathrm{dL}$ $(2.6 \mathrm{mmol} / \mathrm{L})$ that has been adopted by some clinicians. ${ }^{10,25-27,39}$ Cornblath et al. ${ }^{10}$ summarized the problem as follows:

Significant hypoglycemia is not and cannot be defined as a single number that can be applied universally to every indi- vidual patient. Rather, it is characterized by a value(s) that is unique to each individual and varies with both their state of physiologic maturity and the influence of pathology

A meta-analysis of studies published from 1986 to 1994 looked at low plasma glucose thresholds in term healthy newborns who were mostly mixed fed (breastfed and formula-fed) or formula-fed. It presented statistical ranges of low thresholds for plasma glucose level based on hours after birth in healthy term infants (Table 1). ${ }^{40}$ The authors specifically noted that given the known lower plasma glucose levels in healthy term breastfed infants as compared with formula-fed infants, the low thresholds for exclusively breastfed infants might even be lower. Table 1 gives recommendations for this timed threshold approach.

This information is translated into guidelines for clinical intervention by the operational treatment guidance of Cornblath et al. ${ }^{10}$ As they stated, an operational threshold is that concentration of plasma or whole blood glucose at which clinicians should consider intervention, based on the evidence currently available in the literature (Table 2). It needs to be underscored that the therapeutic objective $(45 \mathrm{mg} / \mathrm{dL}[2.5 \mathrm{mmol} / \mathrm{L}])$ is different from the operational threshold for intervention $(36 \mathrm{mg} / \mathrm{dL}$ $[2.0 \mathrm{mmol} / \mathrm{L}]$ ), which is different from the population low thresholds in normal babies with no clinical signs or risk factors who do not need to be treated (Table 1). The higher therapeutic goal was chosen to include a significant margin of safety in the absence of data evaluating the correlation between glucose levels in this range and long-term outcome in full-term infants. ${ }^{10}$

Given this information, it is clear that routine monitoring of blood glucose in healthy term infants is not only unnecessary, but is instead potentially harmful to the establishment of a healthy mother-infant relationship and successful breastfeeding patterns. ${ }^{1,20,22,23,41,42}$ This recommendation has been supported by the World Health Organization, ${ }^{18}$ the American Academy of Pediatrics, ${ }^{1,41}$ the U.S. National Institutes of Health, ${ }^{26}$ and the National Childbirth Trust of the United Kingdom. ${ }^{43}$ These organizations all conclude that (1) early and exclusive breastfeeding is safe to meet the nutritional needs of healthy term infants and that (2) healthy term

Table 2. Operational Thresholds for Treatment of Plasma Glucose Levels ${ }^{10}$

\begin{tabular}{|c|c|c|}
\hline Infant & Plan/PGL & Treatment \\
\hline $\begin{array}{l}\text { Infant with clinical } \\
\text { signs }\end{array}$ & $\begin{array}{l}\text { If }<45 \mathrm{mg} / \mathrm{dL} \\
\quad(2.5 \mathrm{mmol} / \mathrm{L})\end{array}$ & $\begin{array}{l}\text { Clinical interventions to increase } \\
\text { blood glucose concentration }\end{array}$ \\
\hline $\begin{array}{l}\text { Infants with } \\
\text { risk factors }\end{array}$ & $\begin{array}{l}\text { Initiate glucose monitoring as } \\
\text { soon as possible after birth, } \\
\text { within } 2-3 \text { hours after birth } \\
\text { and before feeding, or at any } \\
\text { time there are abnormal signs. } \\
\text { If plasma glucose concentration } \\
\text { is }<36 \mathrm{mg} / \mathrm{dL}(2.0 \mathrm{mmol} / \mathrm{L}) \\
\text { close surveillance should } \\
\text { be maintained. Intervention } \\
\text { is recommended if plasma } \\
\text { glucose remains below this level, } \\
\text { does not increase after a feed, or } \\
\text { if abnormal clinical signs develop. }\end{array}$ & $\begin{array}{l}\text { Clinical interventions to increase blood glucose } \\
\text { concentration: at very low glucose concentration } \\
\text { ( } 20-25 \mathrm{mg} / \mathrm{dL}, 1.1-1.4 \mathrm{mmol} / \mathrm{L}) \text {, intravenous } \\
\text { glucose infusion to raise plasma glucose levels } \\
\text { to }>45 \mathrm{mg} / \mathrm{dL}(2.5 \mathrm{mmol} / \mathrm{L}) \text { is indicated. }\end{array}$ \\
\hline
\end{tabular}

PGL, plasma glucose level.

${ }^{\mathrm{a}}$ See Table 3. 
infants do not develop clinically significant hypoglycemia simply as a result of a time-limited duration of underfeeding.

\section{Testing methods}

Bedside glucose reagent test strips are inexpensive and practical but are not reliable, with significant variance from true blood glucose levels, especially at low glucose concentrations. ${ }^{22,38,44-46}$ Bedside glucose tests may be used for screening, but laboratory levels sent STAT (immediate determination, without delay) (e.g., glucose oxidase, hexokinase, or dehydrogenase method) must confirm results before a diagnosis of hypoglycemia can be made, especially in infants with no clinical signs. ${ }^{1,18,22}$ Other bedside rapid measurement methods such as reflectance colorimetry and electrode methods may be more accurate. ${ }^{47-50}$ Continuous subcutaneous glucose monitoring, as is used in diabetic patients, has been used experimentally in neonates with good correlation with laboratory glucose values but is not currently recommended for screening. ${ }^{51,52}$

\section{Risk factors for hypoglycemia}

Neonates at increased risk for developing neonatal hypoglycemia should be routinely monitored for blood glucose levels irrespective of the mode of feeding. At-risk neonates fall into two main categories:

1. Excess utilization of glucose, which includes the hyperinsulinemic states

2. Inadequate production or substrate delivery ${ }^{32,53,54}$

Infant risk factors for hypoglycemia are listed in Table 3. 3,10,18,19,21,30,32,34,53-56

\section{Clinical manifestations of hypoglycemia}

The clinical manifestations of hypoglycemia are nonspecific, occurring with various other neonatal problems. Even in the presence of an arbitrary low glucose level, the physician must assess the general status of the infant by observation and physical examination to rule out other disease entities and processes that may need additional laboratory evaluation and treatment. Some common clinical signs are listed in Table 4.

A recent study found that of the 23 maternal/infant risk factors and infant signs/symptoms studied, only jitteriness and tachypnea were statistically significant at predicting low blood glucose - not even maternal diabetes! $!^{57}$ A diagnosis of hypoglycemia also requires that signs abate after normoglycemia is restored (the exception being if brain injury has already been sustained).

\section{General Management Recommendations (Table 5)}

Any approach to management needs to account for the overall metabolic and physiologic status of the infant and should not unnecessarily disrupt the mother-infant relationship and breastfeeding. ${ }^{1,21}$ Because severe, prolonged hypoglycemia with clinical signs may result in neurologic injury, ${ }^{11,14,15,58}$ immediate intervention is needed for infants with clinical signs. Several authors have suggested algorithms for screening and treatment. ${ }^{1,17,26,27,59}$ (Quality of evidence [levels of evidence I, II-1, II-2, II-3, and III] is based on the U.S. Preventive Services Task Force Appendix A Task Force Ratings ${ }^{60}$ and is noted in parentheses.)
TABle 3. At-Risk Infants For Whom Routine Monitoring of Blood Glucose Is Indicated

Small for gestational age: $<10^{\text {th }}$ percentile for weight commonly cited in the United States; $<2^{\text {nd }}$ percentile cited in the United Kingdom as above this considered small normal $^{\mathrm{a}}$

Babies with clinically evident wasting of fat and muscle bulk

LGA: $>90^{\text {th }}$ percentile for weight and macrosomic appearance $^{\mathrm{b}}$

Discordant twin: weight $10 \%<$ larger twin

All infants of diabetic mothers, especially if poorly controlled

Low birth weight $(<2,500 \mathrm{~g})$

Prematurity $(<35$ weeks, or late preterm infants with clinical signs or extremely poor feeding)

Perinatal stress: severe acidosis or hypoxia-ischemia

Cold stress

Polycythemia (venous Hct $>70 \%$ )/hyperviscosity

Erythroblastosis fetalis

Beckwith-Wiedemann's syndrome

Microphallus or midline defect

Suspected infection

Respiratory distress

Known or suspected inborn errors of metabolism or endocrine disorders

Maternal drug treatment (e.g., terbutaline, beta-blockers, oral hypoglycemics)

Infants displaying signs associated with hypoglycemia (see Table 4)

\footnotetext{
${ }^{\mathrm{a}}$ As per Dr. Jane Hawdon (personal communication).

${ }^{\mathrm{b}}$ Unnecessary to screen all large for gestational age (LGA) babies. Glucose monitoring is recommended for infants from maternal populations who were unscreened for diabetes during the pregnancy where LGA may represent undiagnosed and untreated maternal diabetes.

Hct, hematorit.
}

\section{A. Initial management}

Early and exclusive breastfeeding meets the nutritional and metabolic needs of healthy, term newborn infants. Healthy term infants do not develop clinically significant hypoglycemia simply as a result of time-limited underfeeding. ${ }^{18,19,21}$ (III)

1. Healthy, appropriate weight for gestational age, term infants should initiate breastfeeding within 30-60 minutes of life and continue breastfeeding on cue, with

\section{Table 4. Clinical Manifestations of Possible} HYPOGLYCEMIA

Irritability, tremors, jitteriness

Exaggerated Moro reflex

High-pitched cry

Seizures or myoclonic jerks

Lethargy, listlessness, limpness, hypotonia

Coma

Cyanosis

Apnea or irregular breathing

Tachypnea

Hypothermia; temperature instability

Vasomotor instability

Poor suck or refusal to feed 
Table 5. General Management Recommendations FOR All TERM INFANTS

A. Early and exclusive breastfeeding meets the nutritional and metabolic needs of healthy, term newborn infants.

1. Routine supplementation is unnecessary.

2. Initiate breastfeeding within 30-60 minutes of life and continue on demand.

3. Facilitate skin-to-skin contact of mother and infant.

4. Feedings should be frequent, 10-12 times per 24 hours in the first few days after birth.

B. Glucose screening is performed only on at-risk infants or infants with clinical signs.

1. Routine monitoring of blood glucose in all term newborns is unnecessary and may be harmful.

2. At-risk infants should be screened for hypoglycemia with a frequency and duration related to the specific risk factors of the individual infant.

3. Monitoring continues until normal, prefeed levels are consistently obtained.

4. Bedside glucose screening tests must be confirmed by formal laboratory testing.

the recognition that that crying is a very late sign of hunger. $^{41,61,62}$ (III)

2. Initiation and establishment of breastfeeding, and reduction of hypoglycemia risk, are facilitated by skinto-skin contact between the mother and her infant immediately after birth for at least the first hour of life and continuing as much as possible. Such practices will maintain normal infant body temperature and reduce energy expenditure (thus enabling maintenance of normal blood glucose) while stimulating suckling and milk production. ${ }^{31,41}$ (II-2, III)

3. Feedings should be frequent, at least 10-12 times per 24 hours in the first few days after birth. ${ }^{41}$ (III) However, it is not unusual for term infants to feed immediately after birth and then sleep quite a long time (up to $8-12$ hours) before they become more active and begin to suckle with increasing frequently. They mount protective metabolic responses throughout this time so it is not necessary to try to force-feed them. However, an unusually, excessively drowsy baby must undergo clinical evaluation.

4. Routine supplementation of healthy term infants with water, glucose water, or formula is unnecessary and may interfere with the establishment of normal breastfeeding and normal metabolic compensatory mechanisms. ${ }^{3,30,41,43}$ (II-2, III)

\section{B. Blood glucose screening}

Glucose screening should be performed only on at-risk infants and those with clinical signs compatible with hypoglycemia. Early breastfeeding is not precluded just because the infant meets the criteria for glucose monitoring.

1. At-risk infants should be screened for hypoglycemia with a frequency and duration related to the specific risk factors of the individual infant. ${ }^{1,19}$ (III) Monitoring should begin no later than 2 hours of age for infants in risk categories. ${ }^{1}$ Hawdon ${ }^{63}$ recommended blood glucose monitoring should commence before the second feeding (i.e., not so soon after birth that the physiologic fall in blood glucose level causes confusion and overtreatment). (III)

2. Monitoring should continue until acceptable, prefeed levels are consistently obtained, meaning until the infant has had at least two consecutive satisfactory measurements. ${ }^{63}$ A reasonable (although arbitrary) goal is to maintain plasma glucose concentrations between 40 and $50 \mathrm{mg} / \mathrm{dL}$ (between 2.2 and $2.8 \mathrm{mmol} / \mathrm{L}$ ) ${ }^{1}$ or $>45 \mathrm{mg} / \mathrm{dL}$ $(2.5 \mathrm{mmol} / \mathrm{L}) .^{10}$ (III)

3. Bedside glucose screening tests must be confirmed by formal laboratory testing, although treatment should begin immediately in infants with clinical signs.

Table 5 summarizes these recommendations.

\section{Management of Documented Hypoglycemia (Table 6)}

A. Infant with no clinical signs (absence of clinical signs can only be determined by careful clinical review)

1. Continue breastfeeding (approximately every 1-2 hours) or feed $1-3 \mathrm{~mL} / \mathrm{kg}$ (up to $5 \mathrm{~mL} / \mathrm{kg}$ ) ${ }^{18}$ of expressed breastmilk or substitute nutrition (pasteurized donor human milk, elemental formulas, partially hydrolyzed formulas, or routine formulas). Glucose water is not suitable because of insufficient energy and lack of protein. Recent reports of mothers with diabetes expressing and freezing colostrum prenatally (beginning at 34-36 weeks of gestation) to have it available after birth to avoid artificial feedings should their infant become hypoglycemic are mixed in terms of association with earlier births, and currently this procedure is not widely recommended. ${ }^{64-68}$ (III)

\section{Table 6. Management of Documented Hypoglycemia}

A. Infant with no clinical signs

1. Continue breastfeeding (approximately every 1-2 hours) or feed $1-5 \mathrm{~mL} / \mathrm{kg}$ of expressed breastmilk or substitute nutrition.

2. Recheck blood glucose concentration before subsequent feedings until the value is acceptable and stable.

3. Avoid forced feedings (see above).

4. If the glucose level remains low despite feedings, begin intravenous glucose therapy.

5. Breastfeeding may continue during intravenous glucose therapy.

6. Carefully document response to treatment.

B. Infant with clinical signs or plasma glucose levels $<20$ $25 \mathrm{mg} / \mathrm{dL}(<1.1-1.4 \mathrm{mmol} / \mathrm{L})$

1. Initiate intravenous $10 \%$ glucose solution with a minibolus.

2. Do not rely on oral or intragastric feeding to correct extreme or clinically significant hypoglycemia.

3. The glucose concentration in infants who have had clinical signs should be maintained at $>45 \mathrm{mg} / \mathrm{dL}$ (>2.5 mmol/L).

4. Adjust intravenous rate by blood glucose concentration.

5. Encourage frequent breastfeeding.

6. Monitor glucose concentrations before feedings while weaning off the intravenous treatment until values stabilize off intravenous fluids.

7. Carefully document response to treatment. 
2. Recheck blood glucose concentration before subsequent feedings until the value is acceptable and stable (usually $>40 \mathrm{mg} / \mathrm{dL}[2.2 \mathrm{mmol} / \mathrm{L}]$ ). If staff is unavailable to check blood glucose and an infant has no clinical signs, breastfeeding should never be unnecessarily delayed while waiting for the blood glucose level to be checked.

3. If the infant is simply worn out and not otherwise ill, nasogastric feeds of human milk can be initiated, watching carefully for signs of intolerance or evidence of significant underlying illness. If the neonate is too ill to suck or enteral feedings are not tolerated, avoid forced oral feedings (e.g., nasogastric tube) and instead begin intravenous (IV) therapy (see below). Such an infant is not normal and requires a careful examination and evaluation in addition to more intensive therapy. Term babies should not be given nasogastric feedings. They are much more likely to fight and aspirate.

4. If the glucose level remains low despite feedings, begin IV glucose therapy and adjust the IV rate by blood glucose concentration. Avoid bolus doses of glucose unless blood glucose is unrecordable or there are severe clinical signs (e.g., seizures or coma). If a bolus dose is given, use $5 \mathrm{mg} / \mathrm{kg}$ of glucose in $10 \%$ dextrose preparation.

5. Breastfeeding should continue during IV glucose therapy when the infant is interested and will suckle. Gradually wean from the IV glucose as the serum glucose level normalizes and feedings increase.

6. Carefully document physical examination, screening values, laboratory confirmation, treatment, and changes in clinical condition (i.e., response to treatment).

7. The infant should not be discharged until reasonable levels of blood glucose are maintained through a fast of 3-4 hours. Monitoring must be recommenced if there are adverse changes in feeding.

\section{B. Infants with clinical signs or with plasma glucose levels $<20-25 \mathrm{mg} / \mathrm{dL}(<1.1-1.4 \mathrm{mmol} / \mathrm{L})$}

1. Initiate IV $10 \%$ glucose solution with a bolus of $3 \mathrm{~mL} / \mathrm{kg}$ and continuous IV treatment at $5-8 \mathrm{mg} / \mathrm{kg} /$ minute.

2. Do not rely on oral or intragastric feeding to correct extreme or symptomatic hypoglycemia. Such an infant most likely has an underlying condition and, in addition to IV glucose therapy, requires an immediate and careful examination and evaluation.

3. The glucose concentration in infants with clinical signs should be maintained at $>45 \mathrm{mg} / \mathrm{dL}(>2.5 \mathrm{mmol} / \mathrm{L})$.

4. Adjust the IV rate by blood glucose concentration.

5. Encourage frequent breastfeeding after initiation of IV therapy.

6. Monitor glucose concentrations before feedings while gradually weaning from the IV solution, until values are stabilized off IV fluids.

7. Carefully document physical examination, screening values, laboratory confirmation, treatment, and changes in clinical condition (i.e., response to treatment).

\section{Supporting the Mother}

Giving birth to an infant who develops hypoglycemia is of concern to both the mother and family and thus may jeop- ardize the establishment of breastfeeding. Mothers should be explicitly reassured that there is nothing wrong with their milk and that supplementation is usually temporary. Having the mother hand-express or pump milk that is then fed to her infant can overcome feelings of maternal inadequacy as well as help establish a full milk supply. It is important for the mother to provide stimulation to the breasts by manual or mechanical expression with appropriate frequency (at least eight times in 24 hours) until her baby is latching and suckling well to protect her milk supply. Keeping the infant at breast or returning the infant to the breast as soon as possible is important. Skin-to-skin care is easily accomplished with an IV line in place and may lessen the trauma of intervention, while also providing physiologic thermoregulation, thus contributing to metabolic homeostasis.

\section{Recommendations for Future Research}

1. Well-planned, well-controlled studies are needed that look at plasma glucose concentrations, clinical signs, and long-term sequelae to determine what levels of blood glucose are the minimum safe levels.

2. The development and implementation of more reliable bedside testing methods would increase the efficiency of diagnosis and treatment of significant glucose abnormalities.

3. Studies to determine a clearer understanding of the role of other glucose-sparing fuels and the methods to measure them in a clinically meaningful way and time frame are required to aid in understanding which babies are truly at risk of neurologic sequelae and thus must be treated.

4. For those infants who do become hypoglycemic, research into how much enteral glucose, and in what form, is necessary to raise blood glucose to acceptable levels is important for clinical management.

5. Randomized controlled studies of prenatal colostrum expression and storage for mothers with infants at risk of hypoglycemia are important to determine if this is a practical and safe treatment modality.

\section{Summary}

Healthy term infants are programmed to make the transition from their intrauterine constant flow of nutrients to their extrauterine intermittent nutrient intake without the need for metabolic monitoring or interference with the natural breastfeeding process. Homeostatic mechanisms ensure adequate energy substrate is provided to the brain and other organs, even when feedings are delayed. The normal pattern of early, frequent, and exclusive breastfeeding meets the needs of healthy term infants.

Routine screening and supplementation are not necessary and may harm the normal establishment of breastfeeding. Current evidence does not support a specific blood concentration of glucose that correlates with signs or that can predict permanent neurologic damage in any given infant. At-risk infants should be screened, followed up as needed, and treated with supplementation or IV glucose if there are clinical signs or suggested thresholds are reached. Bedside screening is helpful, but not always accurate, and should be confirmed with laboratory glucose measurement. A single low glucose value is not associated with long-term neurological abnormalities, 
provided the treating clinician can be assured that the baby was entirely well up until the time of the low value. Hypoglycemic encephalopathy and poor long-term outcome are extremely unlikely in infants with no clinical signs and are more likely in infants who manifest clinical signs and/or with persistent or repeated episodes of severe hypoglycemia.

\section{Acknowledgments}

This work was supported in part by a grant from the Maternal and Child Health Bureau, U.S. Department of Health and Human Services.

\section{References}

1. Adamkin DH, Committee on Fetus and Newborn. Postnatal glucose homeostasis in late-preterm and term infants. $P e$ diatrics 2011;127:575-579.

2. Srinivasan G, Pildes RS, Cattamanchi G, et al. Plasma glucose values in normal neonates: A new look. $J$ Pediatr 1986;109:114-117.

3. Hawdon JM, Ward Platt MP, Aynsley-Green A. Patterns of metabolic adaptation for preterm and term infants in the first neonatal week. Arch Dis Child 1992;67(4 Spec No): 357-365.

4. Cornblath M, Reisner SH. Blood glucose in the neonate and its clinical significance. N Engl J Med 1965;273:378-381.

5. Heck LJ, Erenberg A. Serum glucose levels in term neonates during the first 48 hours of life. J Pediatr 1987;110: $119-122$.

6. Hoseth E, Joergensen A, Ebbesen F, et al. Blood glucose levels in a population of healthy, breast fed, term infants of appropriate size for gestational age. Arch Dis Child Fetal Neonatal Ed 2000;83:F117-F119.

7. Lucas A, Boyes S, Bloom SR, et al. Metabolic and endocrine responses to a milk feed in six-day-old term infants: Differences between breast and cow's milk formula feeding. Acta Paediatr Scand 1981;70:195-200.

8. Edmond J, Auestad N, Robbins RA, et al. Ketone body metabolism in the neonate: Development and the effect of diet. Fed Proc 1985;44:2359-2364.

9. Yager JY, Heitjan DF, Towfighi J, Vannucci RC. Effect of insulin-induced and fasting hypoglycemia on perinatal hypoxic-ischemic brain damage. Pediatr Res 1992;31:138-142.

10. Cornblath M, Hawdon JM, Williams AF, et al. Controversies regarding definition of neonatal hypoglycemia: Suggested operational thresholds. Pediatrics 2000;105: 1141-1145.

11. Boluyt N, van Kempen A, Offringa M. Neurodevelopment after neonatal hypoglycemia: A systematic review and design of an optimal future study. Pediatrics 2006;117:2231-2243.

12. Koivisto M, Blanco-Sequeiros M, Krause U. Neonatal symptomatic and asymptomatic hypoglycaemia: A follow-up study of 151 children. Dev Med Child Neurol 1972;14:603-614.

13. Kinnala A, Rikalainen H, Lapinleimu H, et al. Cerebral magnetic resonance imaging and ultrasonography findings after neonatal hypoglycemia. Pediatrics 1999;103:724-729.

14. Dalgic N, Ergenekon E, Soysal S, et al. Transient neonatal hypoglycemia-Long-term effects on neurodevelopmental outcome. J Pediatr Endocrinol Metab 2002;15:319-324.

15. Burns C, Rutherford M, Boardman J, et al. Patterns of cerebral injury and neurodevelopmental outcomes after symptomatic neonatal hypoglycemia. Pediatrics 2008;122: $65-74$.
16. Menni F, deLonlay P, Sevin C, et al. Neurologic outcomes of 90 neonates and infants with persistent hyperinsulinemic hypoglycemia. Pediatrics 2001;107:476-479.

17. Rozance PJ, Hay WW Jr. Describing hypoglycemiaDefinition or operational threshold? Early Hum Dev 2010;86: 275-280.

18. Williams AF. Hypoglycemia of the Newborn: Review of the Literature. World Health Organization, Geneva, 1997.

19. Eidelman AI. Hypoglycemia and the breastfed neonate. Pediatr Clin North Am 2001;48:377-387.

20. Hawdon JM, Ward Platt MP, Aynsley-Green A. Prevention and management of neonatal hypoglycaemia. Arch Dis Child Fetal Neonatal Ed 1994;70:F60-F64; discussion F65.

21. Wight N. Hypoglycemia in breastfed neonates. Breastfeed Med 2006;1:253-262.

22. Hawdon JM, Platt MP, Aynsley-Green A. Neonatal hypoglycaemia-Blood glucose monitoring and baby feeding. Midwifery 1993;9:3-6.

23. Hawdon J. Neonatal hypoglycemia: The consequences of admission to the special care nursery. Child Health 1993; (Feb): 48-51.

24. Kalhan S, Peter-Wohl S. Hypoglycemia: What is it for the neonate? Am J Perinatol 2000;17:11-18.

25. Sinclair JC. Approaches to the definition of neonatal hypoglycemia. Acta Paediatr Jpn 1997;39(Suppl 1):S17S20.

26. Hay WW, Raju T, Higgens R, et al. Knowledge gaps and research needs for understanding and treating neonatal hypoglycemia: Workshop report from Eunice Kennedy Shriver National Institute of Child Health and Human Development. J Pediatr 2009;155:612-617.

27. Rozance PJ, Hay WW. Hypoglycemia in newborn infants: Features associated with adverse outcomes. Biol Neonate 2006;90:74-86.

28. Aynsley-Green A. Glucose: A fuel for thought! J Paediatr Child Health 1991;27:21-30.

29. Cornblath M, Schwartz R. Hypoglycemia in the neonate. J Pediatr Endocrinol 1993;6:113-129.

30. Swenne I, Ewald U, Gustafsson J, et al. Inter-relationship between serum concentrations of glucose, glucagon and insulin during the first two days of life in healthy newborns. Acta Paediatr 1994;83:915-919.

31. Durand R, Hodges $S$, LaRock $S$, et al. The effect of skin-toskin breast-feeding in the immediate recovery period on newborn thermoregulation and blood glucose values. Neonatal Intensive Care 1997;10:23-29.

32. Cornblath M, Ichord R. Hypoglycemia in the neonate. Semin Perinatol 2000;24:136-149.

33. Sexson WR. Incidence of neonatal hypoglycemia: A matter of definition. J Pediatr 1984;105:149-150.

34. Harris DL, Weston PJ, Harding JE. Incidence of neonatal hypoglycemia in babies identified as at risk. J Pediatr 2012;161:787-791.

35. Cole MD, Peevy K. Hypoglycemia in normal neonates appropriate for gestational age. J Perinatol 1994;14:118-120.

36. Stanley CA, Baker L. The causes of neonatal hypoglycemia. N Engl J Med 1999;340:1200-1201.

37. Schwartz RP. Neonatal hypoglycemia: How low is too low? J Pediatr 1997;131:171-173.

38. Alkalay A, Klein A, Nagel R, et al. Neonatal nonpersistent hypoglycemia. Neonatal Intensive Care 2001;14:25-34.

39. McGowan JE. Commentary: Neonatal hypoglycemiaFifty years later, the questions remain the same. Neoreviews 2004;5:e363-e364. 
40. Alkalay AL, Sarnat HB, Flores-Sarnat L, et al. Population meta-analysis of low plasma glucose thresholds in full-term normal newborns. Am J Perinatol 2006;23:115-119.

41. Section on Breastfeeding. Breastfeeding and the use of human milk. Pediatrics 2012;129:e827-e841.

42. Haninger NC, Farley CL. Screening for hypoglycemia in healthy term neonates: Effects on breastfeeding. J Midwifery Womens Health 2001;46:292-301.

43. National Childbirth Trust, United Kingdom. Hypoglycemia of the newborn: Guidelines for the appropriate blood glucose screening of breast-fed and bottle-fed babies in the UK. Midwives 1997;110:248-249.

44. Ho HT, Yeung WK, Young BW. Evaluation of "point of care" devices in the measurement of low blood glucose in neonatal practice. Arch Dis Child Fetal Neonatal Ed 2004;89:F356-F359.

45. Altimier L, Roberts W. One Touch II hospital system for neonates: Correlation with serum glucose values. Neonatal Netw 1996;15(2):15-18.

46. Hussain K, Sharief N. The inaccuracy of venous and capillary blood glucose measuurement using reagent strips in the newborn period and the effect of haematocrit. Early Hum Dev 2000;57:111-121.

47. Ellis M, Manandhar DS, Manandhar N, et al. Comparison of two cotside methods for the detection of hypoglycaemia among neonates in Nepal. Arch Dis Child Fetal Neonatal Ed 1996;75:F122-F125.

48. Dahlberg M, Whitelaw A. Evaluation of HemoCue blood glucose analyzer for the instant diagnosis of hypoglycaemia in newborns. Scand J Clin Lab Invest 1997;57:719-724.

49. Sharief N, Hussein K. Comparison of two methods of measurement of whole blood glucose in the neonatal period. Acta Paediatr 1997;86:1246-1252.

50. Schlebusch H, Niesen M, Sorger M, et al. Blood glucose determinations in newborns: Four instruments compared. Pediatr Pathol Lab Med 1998;18:41-48.

51. Harris D, Weston P, Williams C, et al. Cot-side electroencephalography monitoring is not clinically useful in the detection of mild neonatal hypoglycemia. $J$ Pediatr 2011;159:755-760.

52. Harris D, Battin M, Weston P, et al. Continuous glucose monitoring in newborn babies at risk of hypoglycemia. $J$ Pediatr 2010;157:198-202.

53. de Lonlay P, Giurgea I, Touati G, e tal. Neonatal hypoglycaemia: Aetiologies. Semin Neonatol 2004;9:49-58.

54. Cowett RM, Loughead JL. Neonatal glucose metabolism: Differential diagnoses, evaluation, and treatment of hypoglycemia. Neonatal Netw 2002;21:9-19.

55. Sunehag AL, Haymond MW. Glucose extremes in newborn infants. Clin Perinatol 2002;29:245-260.

56. Kalhan S, Parmimi P. Gluconeogenesis in the fetus and neonate. Semin Perinatol 2000;24:94-106.

57. Hoops D, Roberts P, VanWinkle E, et al. Should routine peripheral blood glucose testing be done for all newborns at birth? MCN 2010;35:264-270.
58. Yager J. Hypoglycemic injury to the immature brain. Clin Perinatol 2002;29:651-674.

59. Jain A, Aggarwal R, Jeeva Sankar M, et al. Hypoglycemia in the newborn. Indian J Pediatr 2010;77:1137-1142.

60. Appendix A Task Force Ratings. Guide to Clinical Preventive Services: Report of the U.S. Preventive Services Task Force, 2nd edition. www.ncbi.nlm.nih.gov/books/ NBK15430 (accessed March 28, 2014).

61. American Academy of Pediatrics, American College of Obstetricians and Gynecologists. Guidelines for Perinatal Care, 6th ed. American Academy of Pediatrics, Elk Grove Village, IL, 2008.

62. World Health Oraganization, UNICEF. Protecting, Promoting and Supporting Breast-Feeding: The Special Role of Maternity Services, A Joint WHO/UNICEF Statement. World Health Organization, Geneva, 1989.

63. Hawdon JM. Neonatal hypoglycemia: Are evidence-based clinical guidelines achievable? Neoreviews 2014;15:e91e98.

64. Cox SG. Expressing and storing colostrum antenatally for use in the newborn period. Breastfeed Rev 2006;14: 11-16.

65. Forster DA, McEgan K, Ford R, et al. Diabetes and antenatal milk expressing: A pilot project to inform the development of a randomised controlled trial. Midwifery 2011;27:209-214.

66. Soltani H, Scott AM. Antenatal breast expression in women with diabetes: Outcomes from a retrospective cohort study. Int Breastfeed J 2012;7:18.

67. Chapman T, Pincombe J, Harris M. Antenatal breast expression: A critical review of the literature. Midwifery 2013;29:203-210.

68. Chapman T, Pincombe J, Harris M, et al. Antenatal breast expression: Exploration and extent of teaching practices amongst International Board Certified Lactation Consultant midwives across Australia. Women Birth 2013; 26:41-48.

ABM protocols expire 5 years from the date of publication. Evidence-based revisions are made within 5 years or sooner if there are significant changes in the evidence.

The Academy of Breastfeeding Medicine Protocol Committee Kathleen A. Marinelli, MD, FABM, Chairperson Maya Bunik, MD, MSPH, FABM, Co-Chairperson Larry Noble MD, FABM, Translations Chairperson Nancy Brent, $M D$

Amy E. Grawey, $M D$

Alison V. Holmes, MD, MPH, FABM Ruth A. Lawrence, MD, FABM

Tomoko Seo, MD, FABM

Julie Scott Taylor, MD, MSc, FABM

For correspondence: abm@bfmed.org 